\title{
Does body weight influences perioperative heat loss in minor procedures? - A prospective audit in 200 patients
}

Carolina Mateus, Maria João Gomes, Jorge Silva, Diogo Miguel

Carla Cavaleiro, Humberto Machado

Centro Hospitalar do Porto

Euroanaesthesia 2016 Afles?

\section{Background and Goal of Study}

Perioperative thermoregulation is affected by body morphology, once the adipose tissue layer prevents dissipation of metabolic heat, so obese patients are most of the time vasodilated, reducing their core-toperipheral temperature gradient.

The goal of this study was to evaluate the relation between perioperative temperature variation (TV) and body weight (BW) in patients submitted to minor procedures.

\section{Material and Methods}

Approval by Hospital Ethics Committee.

Prospective audit - 200 patients submitted to minor surgery between March and July of 2015.

$\checkmark$ Collected data: gender, age, American Society of Anesthesiologists Physical Status (ASA), Body Mass Index (BMI), type of anaesthesia, duration of surgery and use of active warming.
Tympanic temperature was monitored using Grado ${ }^{\circledR}$ thermometer (RM205V model), on five moments:

- Operating Room arrival (T1)

- After anaesthetic induction (T2)

- End of surgery (T3)

- Post Anaesthesia Care Unit arrival (PACU) (T4)

- PACU discharge (T5)

Statistical analysis: number, percentage, mean \pm standard deviation (SD) and median of $\Delta$ T3-T2, $\Delta$ T5-T1 and $\Delta$ T5-T4 were calculated;Mann-Witney $U$ test and Spearman correlation test were used $(p<0,05)\left(S P S S^{\circledR}\right.$ V. $\left.^{22}\right)$.

\section{Results and Discussion}

\begin{tabular}{|c|c|}
\hline Gender & $55 \%$ female \\
\hline Age & 19-92 years (median 61) \\
\hline BMI (mean) & $27,12 \pm 4,23 \mathrm{Kg} / \mathrm{m}^{2}$ \\
\hline Obese $\left(\mathrm{BMI}>30 \mathrm{Kg} / \mathrm{m}^{2}\right)(\mathrm{n})$ & $44(22 \%)$ \\
\hline ASA I / II / III / IV (n) & $8 / 69$ / 22 / 1 \\
\hline Type of anesthesia & $G A-85 \%$ \\
\hline $\begin{array}{l}\text { Duration of surgery (mean } \pm \\
\text { SD) }\end{array}$ & $55 \pm 32$ minutes \\
\hline Active warming & $25 \%$ \\
\hline $\begin{array}{r}29 \\
35\end{array}$ & $\begin{array}{l}\text { rgery } \\
\text { oaroscopic cholecystectomy } \\
\text { ologic endoscopic surgery } \\
\text { yroidectomy } \\
\text { rnioplasty } \\
\text { ctological surgery }\end{array}$ \\
\hline
\end{tabular}

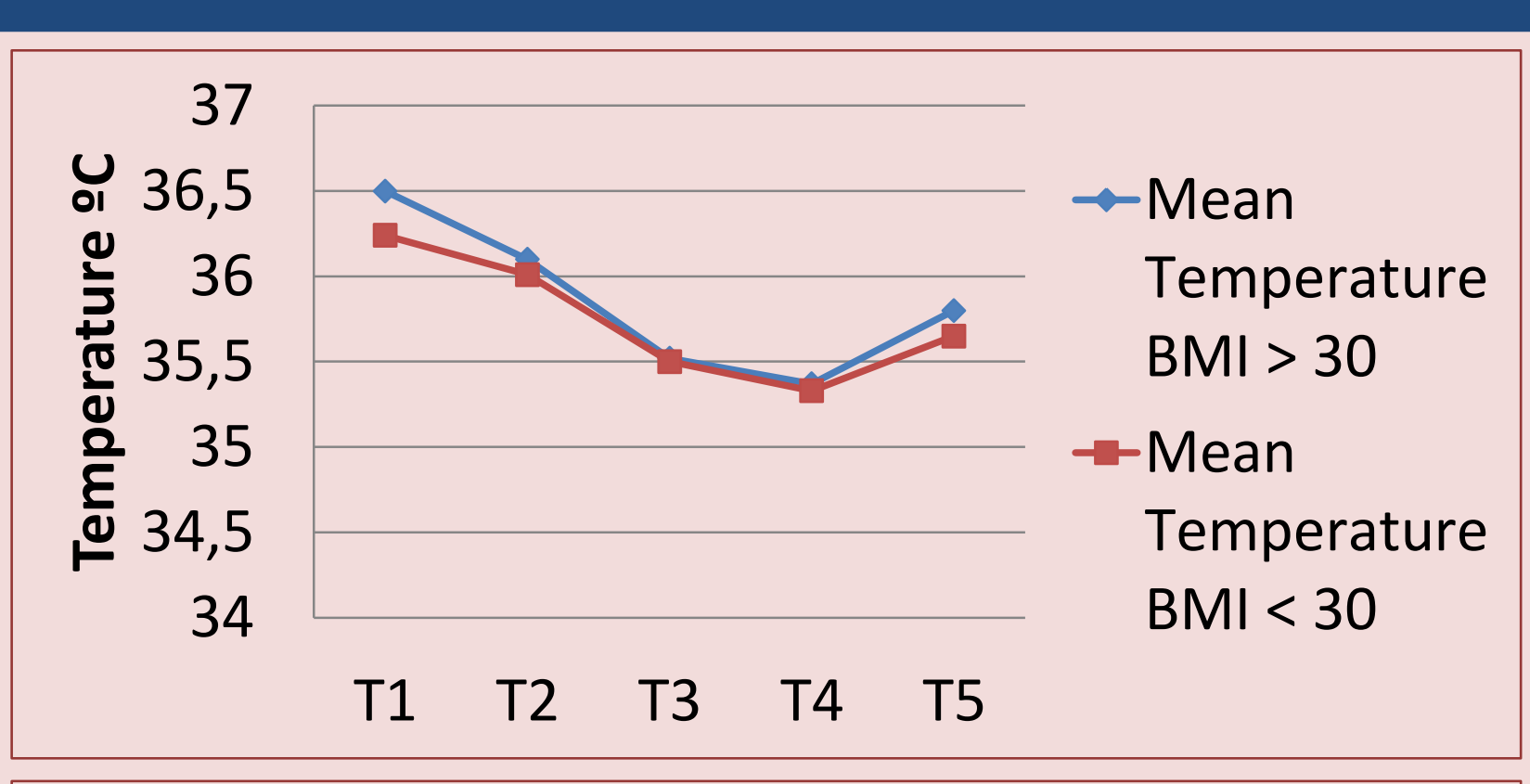

\begin{tabular}{|c|c|c|c|}
\hline \multicolumn{4}{|c|}{ Temperature Variation } \\
\hline \multirow{5}{*}{$\begin{array}{r}1 \\
0,5 \\
0 \\
-0,5 \\
-1 \\
-1,5\end{array}$} & \multirow{2}{*}{\multicolumn{3}{|c|}{1}} \\
\hline & & & \\
\hline & \multirow{2}{*}{\multicolumn{2}{|c|}{1}} & \\
\hline & & & \\
\hline & $\Delta \mathrm{T} 3-\mathrm{T} 2$ & $\Delta \mathrm{T} 5-\mathrm{T} 1$ & $\Delta \mathrm{T} 5-\mathrm{T} 4$ \\
\hline $\mathrm{TV}+\mathrm{SD}$ & $-0,18$ & $-0,07$ & 0,76 \\
\hline TV - SD & $-1,3$ & $-1,09$ & $-0,1$ \\
\hline$\Delta T V$ & $-0,74$ & $-0,58$ & 0,33 \\
\hline
\end{tabular}

$\checkmark$ No significant relation was found between TV and BW during perioperative period.

$\checkmark$ No difference between obese and non-obese patients in $\Delta \mathrm{T} 3-\mathrm{T} 2(\mathrm{p}=0,52), \Delta \mathrm{T} 5-\mathrm{T} 1(\mathrm{p}=0,38)$ and $\Delta \mathrm{T} 5-\mathrm{T} 4(\mathrm{p}=0,68)$.

\section{Conclusion}

In our prospective audit we didn't identify any relation between BW and heat loss during the perioperative period and we didn't find differences between obese and non-obese patients and heat loss.

This results are different from data published; more studies are needed to clarify our findings. 